\title{
Twisted link theory
}

\author{
MARIO O BOURGOIN
}

\begin{abstract}
We introduce stable equivalence classes of oriented links in orientable three-manifolds that are orientation $I$-bundles over closed but not necessarily orientable surfaces. We call these twisted virtual links and show that they subsume the virtual knots introduced by L Kauffman and the projective links introduced by Yu V Drobotukhina. We show that these links have unique minimal genus three-manifolds. We use link diagrams to define an extension of the Jones polynomial for these links and show that this polynomial fails to distinguish two-colorable links over nonorientable surfaces from non-two-colorable virtual links.
\end{abstract}

57M25, 57M27, 57M15, 57M05

\section{Introduction}

\subsection{Virtual links}

In 1996, Louis Kauffman introduced a generalization of classical links to stable embeddings of a disjoint union of circles in a thickened compact oriented surface by using the notion of an oriented Gauss code [12]. An example of such a link is shown in Figure 1. Kauffman was motivated in part by the desire to allow all oriented Gauss codes to be

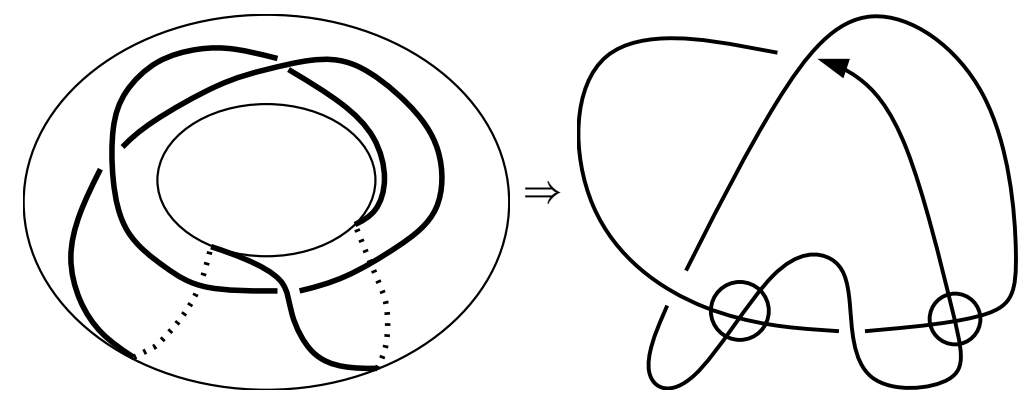

Figure 1: A knot in a thickened torus and its diagram

associated with diagrams of links. When the surface being thickened is a sphere, the links are classical, however there are virtual links that are not classical links. Since by 
a result of Kuperberg [13], virtual links have unique irreducible representatives, virtual link theory is a proper extension of classical links.

Many invariants of classical links formally extend to virtual links through the use of their diagrams by ignoring the virtual crossings, although the resulting invariant may have different properties than those of the classical version. A virtual link can be formally associated with a group through a Wirtinger presentation obtained from any diagram for the link. However, the group of a virtual link may not be residually finite, so it may not be the fundamental group of any three-manifold [18]. A virtual link has a Jones polynomial, but the classical relation between the polynomial's exponents and the number of link components only holds if the link has a two-colorable diagram as was shown by N Kamada [9].

\subsection{Projective links}

In 1990, Yu V Drobotukhina introduced the study of links in real projective space as a generalization of links in the three-sphere [3]. On the left of Figure 2, a nontrivial

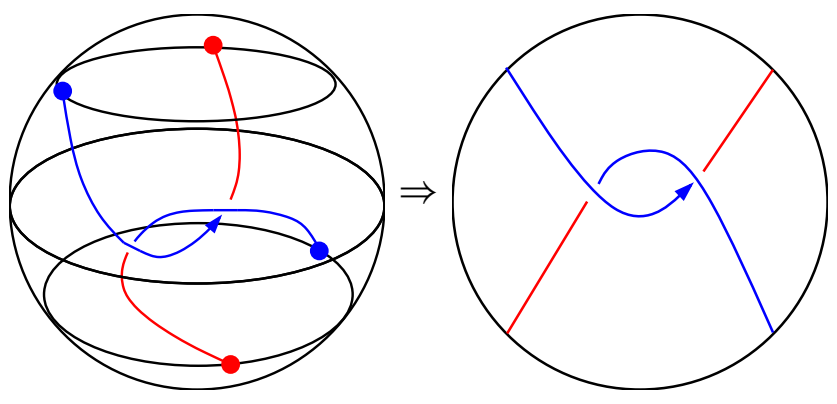

Figure 2: A projective knot and its diagram

projective link is shown using the 3-ball model of projective space. Drobotukhina showed how to create diagrams of projective links in 2-disks, and she extended Reidemeister moves on planar diagrams to include moves across the boundary of the disks. On the right of Figure 2 is shown a diagram of the link in the projective plane. Drobotukhina showed that projective links admit a Jones polynomial invariant and studied some of its properties.

\subsection{Links in oriented thickenings}

We introduce links in oriented thickenings as stable ambient isotopy classes of oriented circles in oriented three-manifolds that are orientation $I$-bundles over closed but not necessarily orientable or connected surfaces. Figure 3 shows a onefoil knot in a 


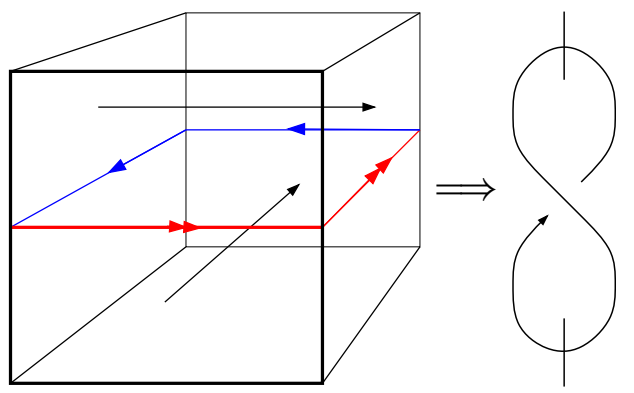

Figure 3: A onefoil knot in a thickened Klein bottle

thickened Klein bottle on the left. The thickened Klein bottle is shown as a cube with identification of its sides with the double arrows with a $180^{\circ}$ turn, and likewise for the sides with the single arrow. A diagram for the onefoil is shown on the right of the figure. An edge of a diagram is an arc between two real crossings. The diagram of the onefoil has an odd number of bars on its edges. Closed paths on the diagram that go over odd-barred edges an odd number of times are orientation-reversing in a projection of the link in the embedded Klein bottle.

By considering destabilization of the oriented thickening along annuli and Möbius bands in the complement of the link, we get an extension to links in oriented thickenings of a result of Greg Kuperberg [13] for virtual links.

Theorem 1 Links in oriented thickenings have a unique irreducible representative.

Thus we may speak of the minimum Euler genus ${ }^{1}$ of a link in oriented thickening. The following is an immediate corollary:

Corollary 1 Links in their minimum Euler genus oriented thickenings that are equivalent through stabilizations are equivalent without stabilizations.

In particular, classical, projective and virtual link theories inject in the theory of link in oriented thickenings.

We define twisted link diagrams as marked generic planar curves, where the markings identify the usual classical crossings, Kauffman's virtual crossings and now bars on edges. We extend the Kauffman-Reidemeister moves for virtual links to account for the new markings by including the twisted moves described in Figure 4. Since there are three classical Reidemeister moves labeled R1 to R3 and four virtual Reidemeister moves labeled V1 to V4, the three twisted Reidemeister moves labeled T1 to T3 


\section{Classical moves}
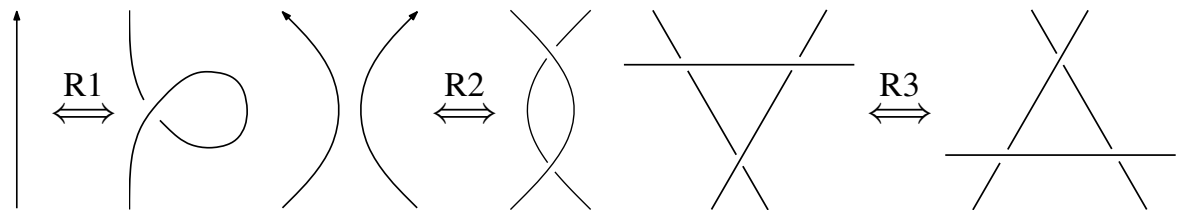

Virtual extension
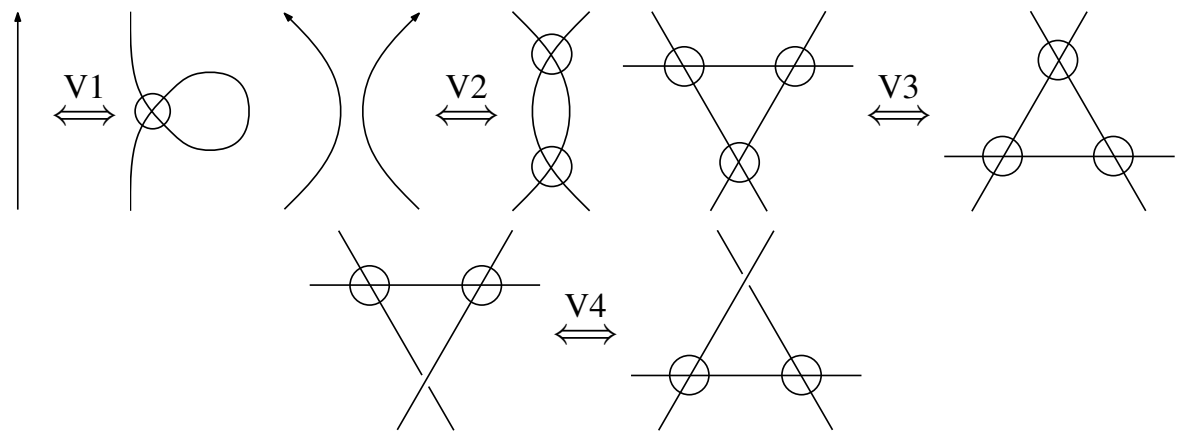

Twisted extension
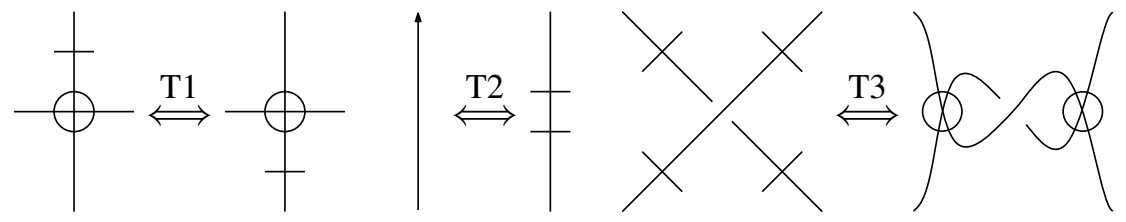

Figure 4: The ten extended Reidemeister moves

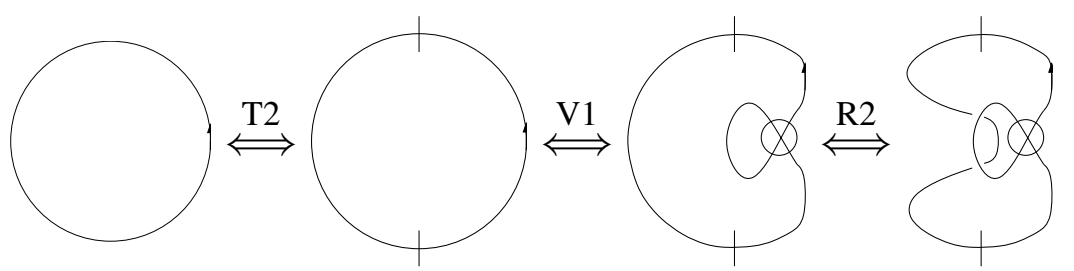

Figure 5: Three extended Reidemeister moves on an unknot

bring the total number of moves to ten. Figure 5 shows an example of transforming the classical diagram of an unknot through each of the three classes of the extended Reidemeister moves. We will see that the leftmost diagram is for an unknot in a thickened sphere while the rightmost diagram is for a curve in a thickened Klein bottle.

\footnotetext{
${ }^{1}$ The Euler genus of a surface is defined as two minus its Euler characteristic.
} 
We have the following:

Theorem 2 Links in oriented thickenings correspond to classes of twisted link diagrams given by ambient isotopy of the diagram along with extended KauffmanReidemeister moves.

Therefore, we will refer to links in oriented thickenings as twisted virtual links or just twisted links.

\subsection{The twisted Jones polynomial}

We use a state sum to define the twisted Jones polynomial of a twisted link as an element of $\mathbb{Z}\left[A^{ \pm 1}, M\right]$, where the $M$ variable counts the number of circles with an odd number of bars in a given state of the diagram. We have the following:

Theorem 3 The twisted Jones polynomial is an invariant of twisted links. If a twisted link diagram is that of a virtual link, then its twisted Jones polynomial is $-A^{-2}-A^{2}$ times its Jones polynomial.

If we let $M=-A^{-2}-A^{2}$ in the twisted Jones polynomial, we can always divide the result by $-A^{-2}-A^{2}$, and then we get an extension of the Jones polynomial to twisted links.

If the polynomial of a twisted link has an $M$ variable, then the link is not a virtual link. It is easy to see that if a link has an odd number of bars on its edges, then we can factor one $M$ variable from its polynomial. On the right of Figure 3 is a onefoil knot in a thickened Klein bottle, and its twisted Jones polynomial is:

$$
V_{\text {Onefoil }}(A, M)=A^{-6}+\left(1-M^{2}\right) A^{-2}
$$

which does not have an $M$ factor. Also its Jones polynomial is trivial.

However, a link may have an $M$-free twisted Jones polynomial and not be virtual, for the knot in Figure 6 has twisted Jones polynomial $\left(-A^{-2}-A^{2}\right)\left(A^{-4}+A^{-6}-A^{-10}\right)$ which is $-A^{-2}-A^{2}$ times its Jones polynomial. This example is noteworthy because while the knot is in a thickening of a projective plane, this diagram in the projective plane is two-colorable. In fact, we have the following.

Theorem 4 If a twisted link has a two-colorable diagram, then its twisted Jones polynomial is $\left(-A^{-2}-A^{2}\right)$ times its Jones polynomial. 


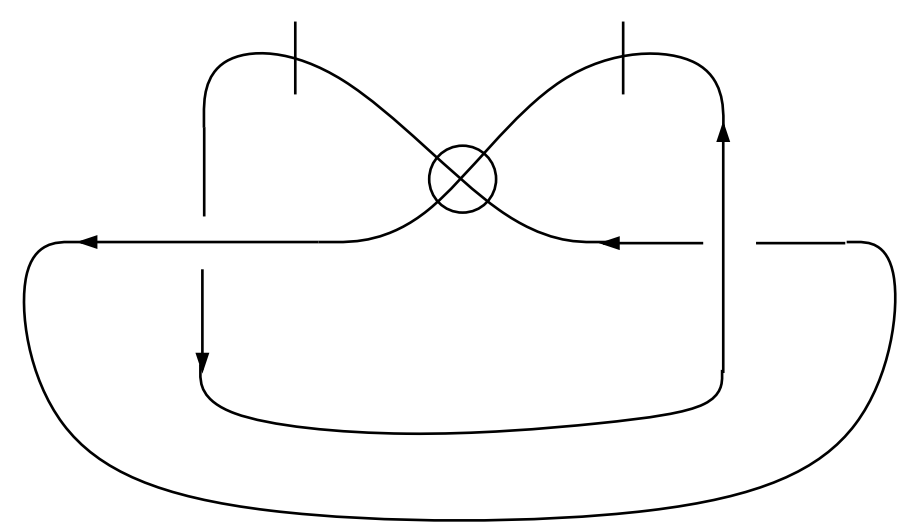

Figure 6: A nonorientable twisted knot

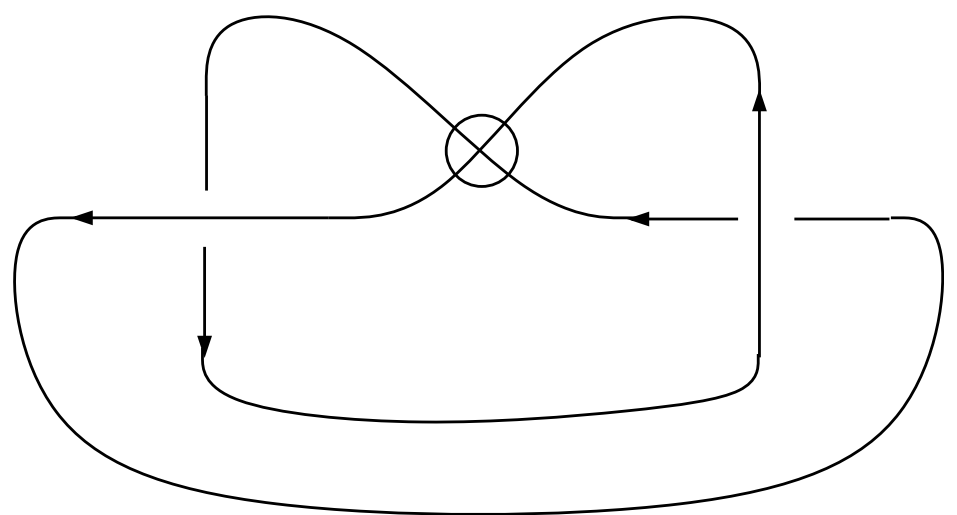

Figure 7: A virtual knot diagram that is not two-colorable

Again, the converse of this theorem is not true since the knot in Figure 7 is a virtual link diagram in a torus and has the same twisted Jones polynomial as the knot in Figure 6, but it does not have a two-colorable diagram because the exponents of its Jones polynomial are not a multiple of four. By a theorem of Naoko Kamada [9], if a virtual link has a two-colorable diagram, then its Jones polynomial's exponents are multiples of four if the link has an odd number of components, and multiples of four plus two if the link has an even number of components.

\subsection{The twisted link group}

Let $L$ be a virtual link. Kauffman defines an invariant of virtual links which he calls the group of the virtual link, $\Pi L$ [12]. This invariant is calculated from any diagram for $L$ by letting the arcs of the diagram between undercrossings be the generators, ignoring 
virtual crossings, and formally applying the Wirtinger algorithm to the diagram to create a presentation of the group. This group is also called the upper group of the virtual link, and when the generators are arcs between overcrossings, the group is called the lower group of the virtual link.

Because the group of a link ignores the bars on the edges, it fails to be invariant with respect to move T3. For example, Figure 8 shows a trefoil on which a T3 move has

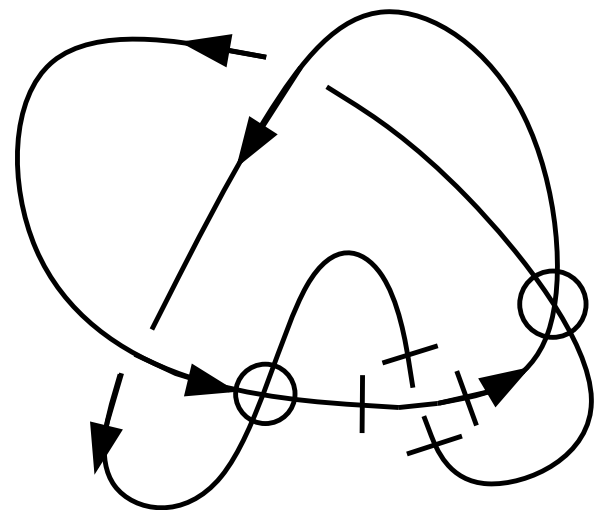

Figure 8: A trefoil under move T3

been performed, and its group is trivial. In this case, we can apply a modified Wirtinger algorithm that defines two generators for every end of an edge in the underlying graph, four relations at every crossing and two relations for every edge, and we define the twisted link group of the link diagram to be the group associated to the presentation. We have the following:

Theorem 5 The twisted link group of a link diagram is an invariant of the link.

It is immediate from the definition of the twisted link group that when a twisted link is virtual, its group is a free product of its upper and lower groups.

The knots in Figure 6 and Figure 7 have identical twisted Jones polynomial, but different twisted link groups. The knot in Figure 6 has a twisted link group with (simplified) presentation:

$$
\widetilde{\Pi}(\text { Twofoil })=\left\langle a, b \mid a^{2}=b^{-1} a^{2} b\right\rangle
$$

which has $\left\langle a^{2}\right\rangle$ in its center. The knot in Figure 7 has a twisted link group which is a free product of its upper and lower groups, and since these are both isomorphic to the integers, the twisted link group has a trivial center. 
However, the twisted link group is not necessarily better than the twisted Jones polynomial at distinguishing knots. The knot in Figure 3 and the unknot have different twisted Jones polynomials, but identical twisted link groups. The twisted link group of the onefoil is a free group on two generators, as is the twisted link group of an unknot. But the twisted Jones polynomial of the onefoil is:

$$
V_{\text {Onefoil }}(A, M)=A^{-6}+\left(1-M^{2}\right) A^{-2} .
$$

So the twisted Jones polynomial and the twisted link group contain different information about the link.

The topological interpretation of the twisted link group is an open problem. In particular, its relationship with the fundamental group of the complement of some realization of the twisted link with collapsed boundary is not as straightforward as in the virtual case (see the author's paper [2]), as some examples have shown that they are not always equal.

\section{Background}

\subsection{Projective links}

Generalizing the study of links in the three-sphere to real projective space, Yu V Drobotukhina [3] created diagrams of projective links in a 2-disk representation of a projective plane and extended Reidemeister moves on planar diagrams to include moves across the boundary of the disks. On the right of Figure 2, is shown a diagram of the link in the projective plane. Figure 9 shows the extension of the Reidemeister
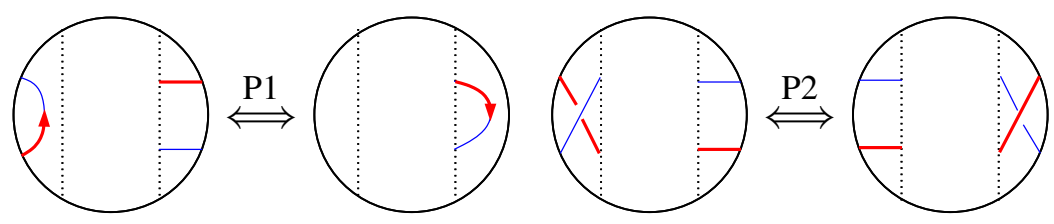

Figure 9: Projective extension of the Reidemeister moves

moves to deal with passing through the boundary of the disk. The strands involved in the moves are shown with different thickness to emphasize the effects of passing through the orientation-reversing boundary of the 2-disk. If a projective link can be isotoped to a link in the affine part of projective space, then it corresponds to a link in the three-sphere. In other papers, Drobotukhina classified nontrivial projective links with diagrams with up to six crossings [5] as well as projective Montesinos links [4]. 


\subsection{Virtual links}

An oriented Gauss code is a double occurrence collection of sequences of symbols with a direction of traversal for each sequence and whose symbols are accompanied by writhe $( \pm)$ and height $(O / U)$ marks with the restriction that both occurrences of the same symbol have the same writhe and different heights. Two oriented Gauss codes over the same symbols are equivalent under permutation of the set of symbols and rotation of the sequences. The string $O 1+U 2+U 1+O 2+$ is an example of a one component Gauss code with two crossings with positive writhe. Kauffman defined a virtual link combinatorially as follows [12, p 668].

A virtual link is an equivalence class of oriented Gauss codes under the abstractly defined Reidemeister moves:

R-1 :

$a b \sim a U 1+O 1+b \sim a U 1-O 1-b \sim a O 1+U 1+b \sim a O 1-U 1-b$

R-2 :

$a O 1-O 2+b U 1-U 2+c \sim a b c \sim a U 1+U 2-b O 1+O 2-c$

$a O 1+O 2-b U 2-U 1+c \sim a b c \sim a U 1-U 2+b O 2+O 1-c$

R-3 :

$a O 1+U 2+b O 3+O 2+c U 1+U 3+d \sim a U 2+O 1+b O 2+O 3+c U 3+U 1+d$

$a O 1+O 2-b O 3+U 2-c U 1+U 3+d \sim a O 2-O 1+b U 2-O 3+c U 3+U 1+d$

$a U 1-O 2-b U 3-U 2-c O 1-O 3-d \sim a O 2-U 1-b U 2-U 3-c O 3-O 1-d$

$a U 1-U 2+b U 3-O 2+c O 1-O 3-d \sim a U 2+U 1-b O 2+U 3-c O 3-O 1-d$

In each move, oriented Gauss codes are presented left-right, the crossing numbers are assigned to reflect the order in which crossings are encountered, and the lowercase letters $a, b, c, \ldots$ represent segments of the code in between which the fragments are embedded.

Kauffman associated every oriented Gauss code with a planar link diagram by the usual graph theory approach of allowing drawings in the plane where some of the crossings are deemed not to occur in the diagram. These crossings are called virtual and do not have writhe or height. A virtual link diagram for Gauss code $O 1+U 2+U 1+O 2+$ is shown in Figure 7. In the diagram, the virtual crossing is circled, and the writhe is obtained by the usual right-hand rule. Kauffman identified an extension of the Reidemeister moves on classical link diagrams to moves on link diagrams with virtual crossings. Some of these are illustrated in Figure 4 where seven of the allowed virtual moves are shown, one for each class of Reidemeister move. Three of the moves involve 
only classical crossings, three of the moves involve only virtual crossings, and one of the moves involves two virtual crossings and one real crossing. Note that the two obvious 3-crossing moves that involve two real crossings and one virtual crossing are forbidden because including them makes all links are equivalent to unlinks by Nelson [17].

Kauffman extended many link invariants, such as the group, quandle, to virtual links by the simple approach of ignoring the virtual crossings.

Abstract links over orientable surfaces were introduced in knot theory by Naoko Kamada and Seiichi Kamada [10]. They are regular neighborhoods of links in surfaces, and are equivalent to virtual links which they anticipated. However, similar presentations for graphs embedded in surfaces have long been common in topological graph theory [6].

\subsection{The Jones polynomial}

In 1985, Vaughan Jones announced the creation of a new polynomial invariant of links [8]. This invariant encoded different information about links than the older Alexander polynomial. This invariant was soon generalized to the HOMFLY polynomial, which generalized both the Jones and Alexander polynomials. In 1987, Louis Kauffman introduced a state-sum model for the calculation of the Jones polynomial [11].

In 1990, Yu V Drobotukhina extended the Jones polynomial to projective links, and generalized results by L Kauffman and K. Murasugi relating the crossing and component numbers of link diagrams to the exponents of the link's Jones polynomial [3]. Drobotukhina used the properties of the Jones polynomial to provide a necessary condition for a projective link to be affine, and characterized the projective links with alternating diagrams that are affine. Recently, M Mroczkowki considered the problem of creating descending diagrams for projective links [15], and used them to extend the HOMFLY and Kauffman polynomials to projective links [16].

In 1996, Louis Kauffman extended the Jones polynomial to virtual links by the simple approach of ignoring the virtual crossings [12]. For example, the bracket polynomial of the virtual link in Figure 7 is $A^{2}+1+A^{-4}$ showing that it is not trivial. Kauffman also showed that his extension does not detect the virtualization of a crossing shown in Figure 10. Soon afterward, the Alexander, HOMFLY, and Kauffman polynomials were also extended to virtual links. In 2002, V O Manturov extended the Jones polynomial so as to detect the virtualization of a crossing [14].

\subsection{The group of a virtual link}

In [12], Louis Kauffman introduced the group of a virtual link as the group defined by the formal Wirtinger presentation obtained from any link diagram by ignoring any 


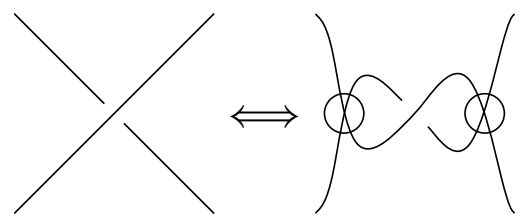

Figure 10: The virtualization of a crossing

virtual crossings. Unlike the classical group of a link, the group of a virtual link can have deficiency zero. More importantly, it may not be residually finite, so it may not be the group of any three-manifold [18], and in particular, it may not be the fundamental group of the complement of the link in any of its stabilized embeddings. Furthermore, the group obtained by defining the generators to be strands between overcrossings may be different from the standard one where generators are strands between undercrossings.

In [10], Naoko Kamada and Seiichi Kamada showed that the group of a virtual knot is the group of a three-complex obtained by collapsing one of the boundary components of the complement of the link in the thickened surface.

\section{Definitions}

The Euler genus of a surface is $2-\chi$ where $\chi$ is the surface's Euler characteristic. A possibly nonorientable closed surface of Euler genus $g$ will be noted $\Sigma_{g}$.

A move of a manifold $M$ is a homeomorphism of $M$ supported by a ball, keeping the boundary of the ball fixed. A standard linear move is a homeomorphism of a standard simplex keeping the boundary fixed, mapping the barycenter to another interior point, and joining linearly. A linear move of $M$ is a move $h$ supported by a ball $B$ for which there exists a homeomorphism $k: B \rightarrow M$ such that $k h k^{-1}$ is a standard linear move. Two embeddings $f, g: N \rightarrow M$ are isotopic by linear moves if there exists a finite sequence $h_{1}, h_{2}, \ldots, h_{n}$ of linear moves such that $h_{1} h_{2} \ldots h_{n} f=g$.

Define the standard interval to be $I=[-1,1]$. An orientable thickening of a closed surface $\Sigma$ is the orientation $I$-bundle $\Sigma \tilde{\times} I$ over the surface. An oriented thickening is an orientable thickening with a given orientation. A link in an oriented thickening is an embedding of a disjoint collection of oriented circles in the interior of an oriented thickening. Two links in an oriented thickening are equivalent if there is an orientationpreserving homeomorphism of the oriented thickening that is isotopic to the identity and takes one link to the other while preserving link orientation.

A band is either an annulus or a Möbius band. A vertical band in an oriented thickening is the fiber over a simple closed path in the zero-section. A vertical annulus is a vertical 
band over an orientation-preserving path, and a vertical Möbius band is a vertical band over an orientation-reversing path. A topologically vertical band is a properly embedded band isotopic to a vertical band.

A destabilization of a link in an oriented thickening consists of cutting the thickening along a vertical band disjoint from the link and capping the resulting boundary components with thickened disks. The result of a destabilization is a link in an oriented thickening descendant from the original link. When the band is a vertical Möbius band, it has a nontrivial normal bundle in the zero-section so its normal bundle in the oriented thickening is trivial. Then the boundary component that remains after cutting along it is an annulus that is capped with a single thickened disk. A stabilization is the reverse of a destabilization. Two destabilizations are descent equivalent if they have equivalent descendants.

A stable link in an oriented thickening is an equivalence class of links in oriented thickenings under (de)stabilizations. Unless explicitly said otherwise, a mention of links in oriented thickenings will always imply equivalence up to stabilization.

A surface projection of a link in an oriented thickening is a regular projection of the link by the bundle projection on the zero-section of the oriented thickening. The components of a surface projection are oriented by the components of the link. Let $\tau, \bar{\tau}$ be unit vectors at a double point each tangent to different parts of the curve through the double point, and that agree with the orientation of the part to which they are tangent. A resolution of a double point of a surface projection is an assignment of unit vectors $\eta, \bar{\eta}$ from the normal bundle to the surface at the double point such that $\eta=-\bar{\eta}$. Figure 11 shows the resolution of a double point. Then the writhe $\mathrm{w}$ at a double point of a generic

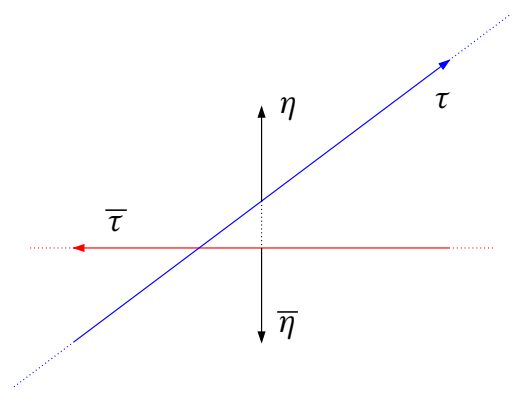

Figure 11: Resolving a double point of a surface projection

immersion is $\mathrm{w}=(\tau \times \bar{\tau}) \cdot \eta=(\bar{\tau} \times \tau) \cdot \bar{\eta}$.

We now consider generic immersions of oriented curves in compact surfaces as in [1]. Let $\tau, \bar{\tau}$ be unit vectors at a double point each tangent to different parts of the curve 
through the double point, and that agree with the orientation of the part to which they are tangent. A separation of a double point of a generic immersion is an assignment of unit vectors $\omega, \bar{\omega}$ at the double point such that $\omega= \pm \tau, \bar{\omega}= \pm \bar{\tau}$ and such that $\tau \cdot \omega=\bar{\tau} \cdot \bar{\omega}$. Then the writhe $\mathrm{w}$ at a double point of a generic immersion is $\mathrm{w}=\tau \cdot \omega$. A link surface diagram is a generic immersion with a separation of each double point. Figure 12 shows a link surface diagram for the onefoil in a Klein bottle with the short fat arrows

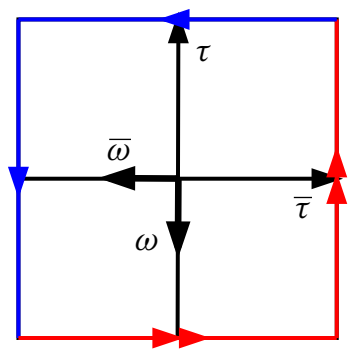

Figure 12: A link surface diagram in a Klein bottle

indicating the separation, in which case, the crossing has -1 writhe. As will be shown later, separations of double points define resolutions of the double points when the surface is the zero-section of an oriented thickening. And unlike the usual over-under knot diagram crossings, they do not need to change if the crossing is isotoped along an orientation-reversing loop in the surface. In drawings of link diagrams in surfaces, the separation can be indicated by the classical over-under convention, so long as an isotopy of a crossing through an orientation-reversing path switches the crossing.

A Reidemeister move on a link surface diagram is one of three basic moves in disk neighborhoods, the cases of which are shown in Figure 13. Two link surface diagrams in homeomorphic surfaces are Reidemeister equivalent if there are sequences of ambient isotopies of the surfaces and Reidemeister moves in disk neighborhoods such that there exists a homeomorphism of the surfaces that carries one diagram onto the other one while preserving the diagrams' orientations.

A destabilization of a link surface diagram consists of cutting the surface along a simple closed path disjoint from the link and capping the resulting boundary components with disks. The result of a destabilization is a link surface diagram descendant from the original link. When the path is orientation-reversing, the boundary component that remains after cutting along it is a circle that is capped with a single disk. A stabilization is the reverse of a destabilization. Two destabilizations are descent equivalent if they have Reidemeister-equivalent descendants. A simple closed path is essential if it does not bound a disk in the complement of a link surface diagram. Figure 14 shows a 

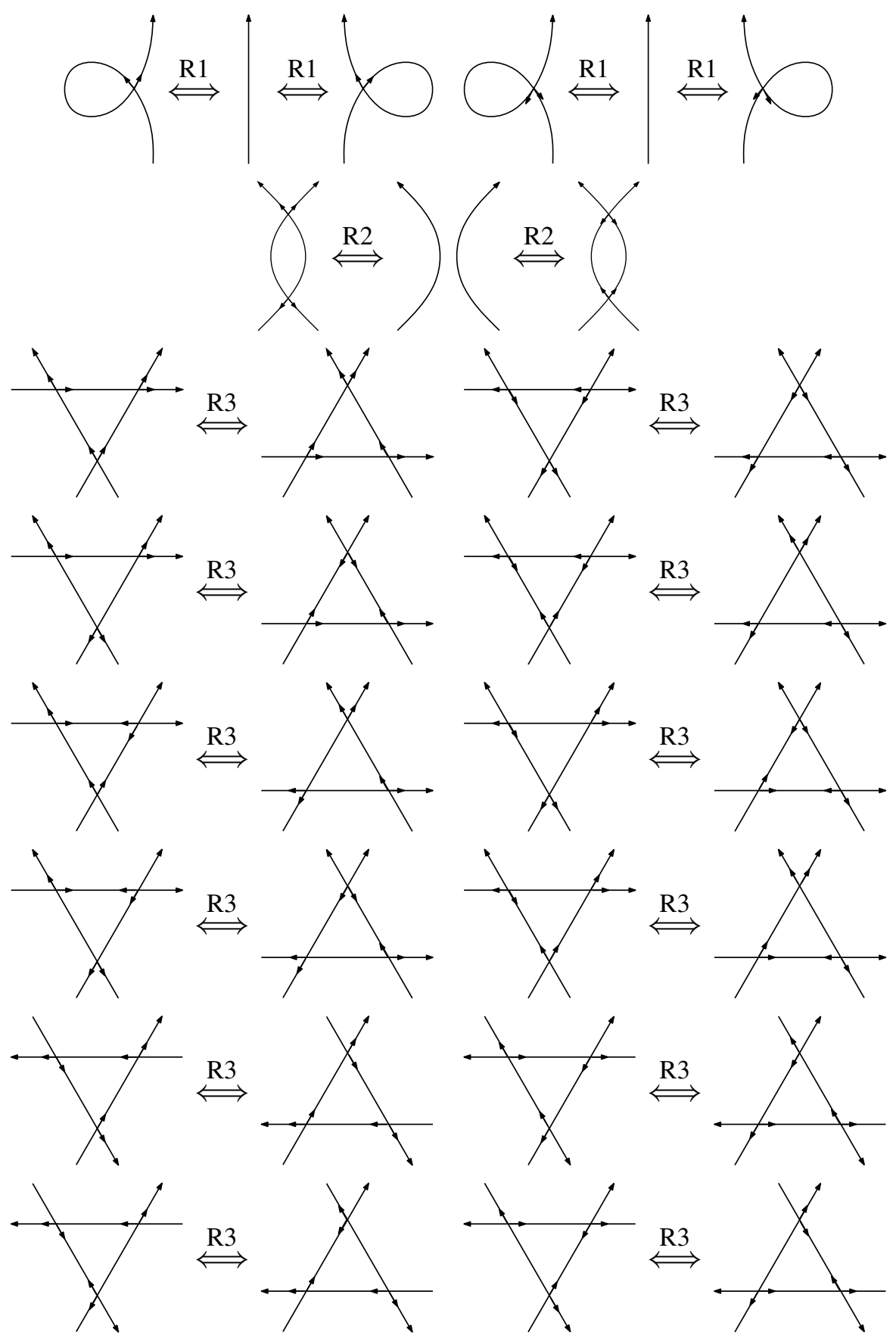

Figure 13: The Reidemeister moves for link surface diagrams 


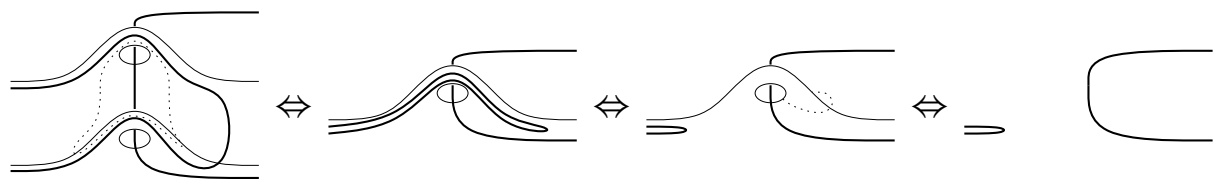

Figure 14: Two surface (de)stabilizations

sequence of two destabilizations along dotted paths that are essential and remove two handles. This is equivalent to performing a V2-move on a virtual link diagram. A stable link surface diagram is an equivalence class of link surface diagrams under (de)stabilizations of the surface that do not intersect the link. Two stable link surface diagrams are stably Reidemeister equivalent if there is a sequence of (de)stabilizations of the surfaces, homeomorphisms of the (de)stabilized surfaces, ambient isotopies of the surfaces, and Reidemeister moves in disk neighborhoods that carries one diagram onto the other one. Unless explicitly said otherwise, "link surface diagram" will always mean stable link surface diagram.

An intersigned link code is a double occurrence collection of sequences of symbols with a direction of traversal for each sequence and whose symbols are accompanied by writhe $( \pm)$ and separated by \pm signs with the restriction that both occurrences of the same symbol have the same writhe. They are in fact intersigned Gauss codes with writhe marks attached to the symbols, and intersigned Gauss codes classify immersed curves in closed compact surfaces [1]. A Reidemeister move on an intersigned link code is one of the abstractly-defined moves:

$$
\begin{array}{ll}
\text { R-1 : } & a b \sim a 1^{\varepsilon}+1^{\varepsilon} b \\
\text { R-2 : } & a 1^{\varepsilon}+2^{-\varepsilon} b 1^{\varepsilon}+2^{-\varepsilon} c \sim a b c \sim a 1^{\varepsilon}+2^{-\varepsilon} b 2^{-\varepsilon}+1^{\varepsilon} c \\
\text { R-3 : } & a 1^{\varepsilon}+2^{\varepsilon} b 3^{\varepsilon}-2^{\varepsilon} c 1^{\varepsilon}-3^{\varepsilon} d \sim a 1^{\varepsilon}+2^{\varepsilon} b 1^{\varepsilon}-3^{\varepsilon} c 3^{\varepsilon}-2^{\varepsilon} d \\
& a 1^{-\varepsilon}+2^{\varepsilon} b 3^{\varepsilon}-2^{\varepsilon} c 1^{-\varepsilon}-3^{\varepsilon} d \sim a 1^{\varepsilon}+2^{-\varepsilon} b 1^{\varepsilon}-3^{\varepsilon} c 3^{\varepsilon}-2^{-\varepsilon} d
\end{array}
$$

In a move, intersigned link codes are presented left-right, the crossing numbers are assigned to reflect the order in which crossings are encountered, the writhe mark is given as an exponent $\varepsilon= \pm$ such that $-\varepsilon=\mp$, and the lowercase letters $a, b, c, \ldots$ represent segments of the code in between which the fragments are embedded. Two intersigned link codes are Reidemeister equivalent if there is a sequence of Reidemeister moves that takes one code to the other.

A ribbon graph is a finite collection of oriented disks with a finite collection of disjoint arcs in their boundaries and (possibly orientation-reversing) homeomorphisms that identify pairs of the boundary arcs such that each boundary arc is identified with exactly one other different boundary arc. Then the boundary of a ribbon graph is a collection 
of disjoint orientation-preserving closed paths called the faces of the ribbon graph. An abstract link is a link surface diagram in the image of an embedding of a ribbon graph in a closed surface such that the link is a deformation retract of the image of the ribbon graph. This definition of an abstract link diagram differs from that of Naoko Kamada and Seiichi Kamada [10] in that in our case, the ribbon graph need not be an orientable surface. Figure 15 shows an abstract link for the onefoil knot in a Klein bottle minus a

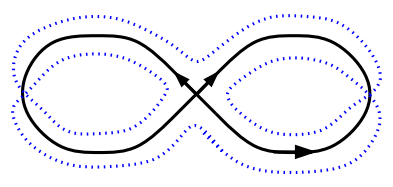

Figure 15: An abstract knot for the onefoil

disk. A Reidemeister move on an abstract link consists of first identifying intervals in the boundary of the ribbon graph to form a disk neighborhood of a collection of edges of the link, performing the Reidemeister move in that disk, and then discarding all but a regular neighborhood of the link. Figure 16 shows an R2 move on an abstract link.

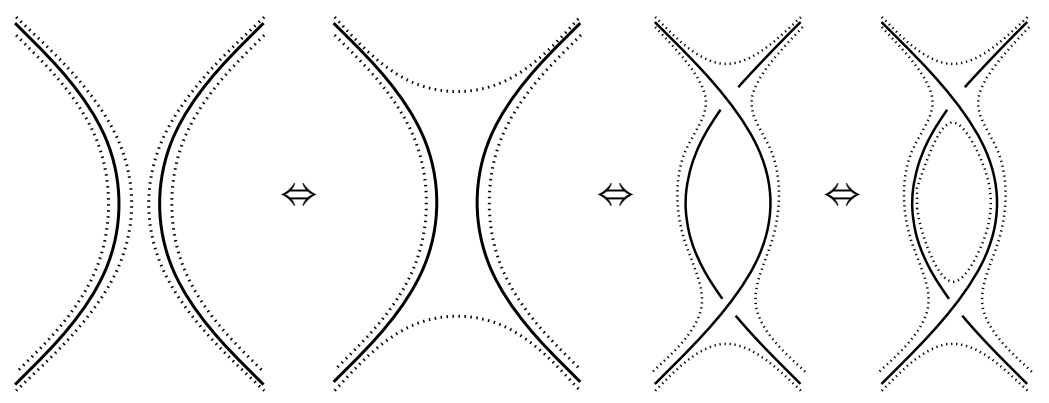

Figure 16: An R2 move on an abstract link

Two abstract links are Reidemeister equivalent if there is a sequence of Reidemeister moves that will make them homeomorphic while preserving link orientation.

A twisted link diagram is a planar immersion of a disjoint union of oriented circles with real and virtual crossings and bar marks on arcs. An edge of a diagram is an arc between two real crossings. Two twisted link diagrams are Reidemeister equivalent if there is a sequence of ambient isotopies of the plane and extended Reidemeister moves that carries one diagram onto the other one. The ten extended Reidemeister moves are diagrammed in Figure 4. The faces of a twisted link diagram are closed curves that run along the immersed curve and have the relationship with the crossings, virtual crossings, and bars as show in Figure 17. At a crossing, a face turns so as to 


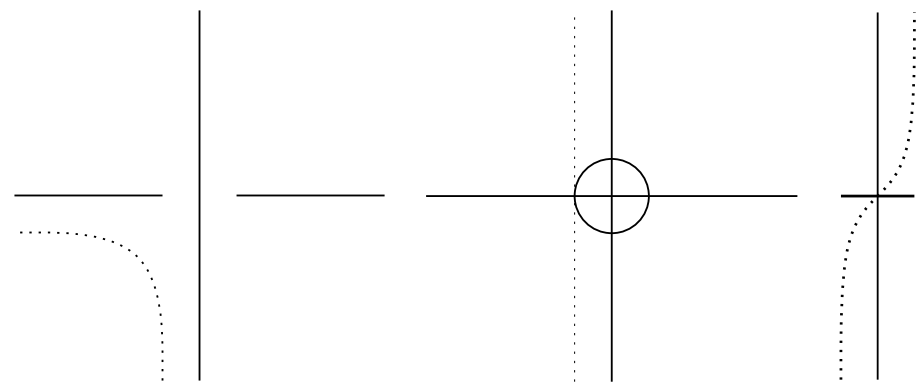

Figure 17: Faces and crossings, virtual crossings, and bars

avoid crossing the link diagram. At a virtual crossing, a face goes through the virtual crossing. At a bar, a face crosses to the other side of the link diagram. A twisted link diagram is two-colorable if its faces can be assigned one of two colors such that the arcs of the link diagram between two crossings always separate faces of one color from those of the other.

\section{Unique destabilization}

According to Greg Kuperberg [13], links in oriented thickenings of orientable closed surfaces have unique irreducible representatives. This is proven by showing that all pairs of destabilizations along essential vertical annuli are descent equivalent to destabilizations along annuli that do not intersect, and these are descent equivalent. Theorem 1 is the equivalent statement for links in oriented thickenings over closed surfaces, and our proof will follow Kuperberg's strategy.

Proof of Theorem 1 We expand the range of surfaces along which destabilizations can occur to include spheres and proper disks that separate some components of the link from the other components, and bands that separate some link-free part of the genus from the surface and so that we may discard that part. The results of such destabilizations can be achieved by destabilizations along vertical bands. In the first case, a disk or sphere may be altered to be a band, and in the second case, the destabilization may be performed along bands that progressively reduce genus but do not increase the number of components of the thickened surface. So we define an admissible surface to be a vertical band, sphere, or proper disk. And an admissible surface is essential if it does not bound a ball in the complement of the link. Then every essential admissible surface can be used for destabilization.

We induct on the complexity of the intersection between two destabilization surfaces. Consider an $n$-component link $L$ in the oriented thickening $M$ of a $c$-component 
surface of Euler genus $g$. We require that every component of $M$ contain at least one component of the link, so $n \geq c$. Suppose that $L$ is a counterexample to the theorem for which $g+2(n-c)$ is minimal. Then $L$ has at least two descent-inequivalent destabilization surfaces, $B_{1}, B_{2}$. Suppose further that these surfaces intersect in the fewest curves amongst such pairs. This intersection is not empty because destabilizations along disjoint surfaces are descent equivalent. Indeed, if the surfaces are isotopic, then destabilization along one or the other produces the same manifold, and otherwise, we may destabilize first along either surface and then along the other to obtain the same manifold. We consider the surfaces to be in general position, so their intersection is an embedded one-manifold, and its components are, up to homotopy, one of the types shown in Figure 18. We deal with each type in turn. We show that using an intersection

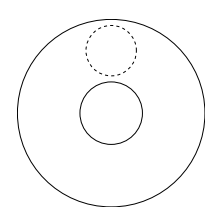

a1

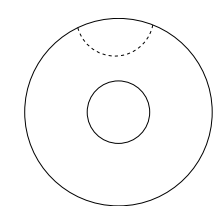

a2

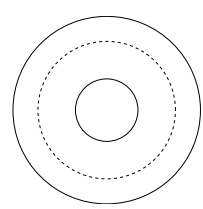

a3

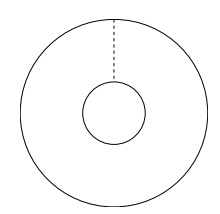

a4

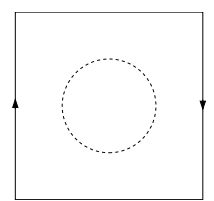

$\mathrm{m} 1$

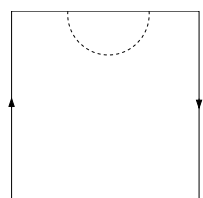

$\mathrm{m} 2$

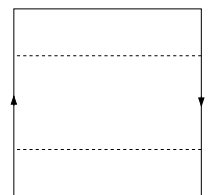

$\mathrm{m} 3$

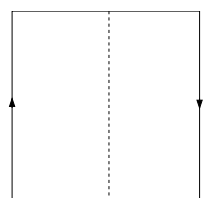

$\mathrm{m} 4$

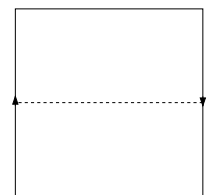

$\mathrm{m} 5$

Figure 18: Types of components of the intersection of two surfaces

component, we can find an essential destabilization surface $B^{\prime}$ whose intersection with $B_{1}$ and $B_{2}$ has fewer curves with either than they have with each other, so it is descent equivalent to both, thus obtaining a contradiction. When $B^{\prime}$ is to be a band constructed from parts of $B_{1}$ and $B_{2}$, we will obtain that it is topologically vertical because it is homotopic to the fiber over a closed path in the zero section made from parts of the closed paths of $B_{1}$ and $B_{2}$.

Cases a1, a2, $\mathbf{m} 1$, and $\mathbf{m} 2$ The curve is an arc or circle in $B_{1}$. Then we can find an innermost curve that bounds a disk that does not contain any other component of the intersection. (In cases $\mathrm{a} 2$ and $\mathrm{m} 2$, the disk is formed with part of the boundary of $B_{1}$.) We may compress $B_{2}$ along this disk to get surfaces $B_{2}^{\prime}, B_{2}^{\prime \prime}$ at least one of which, say $B_{2}^{\prime}$ is an admissible essential surface. In Figure 19 the left image shows the intersection of $B_{1}$ with $B_{2}$, while the right image shows the constructed $B_{2}^{\prime}$ and $B_{2}^{\prime \prime}$ in thick lines. Since $B_{2}^{\prime}$ intersects $B_{1}$ less than does $B_{2}$, then it is descent equivalent 


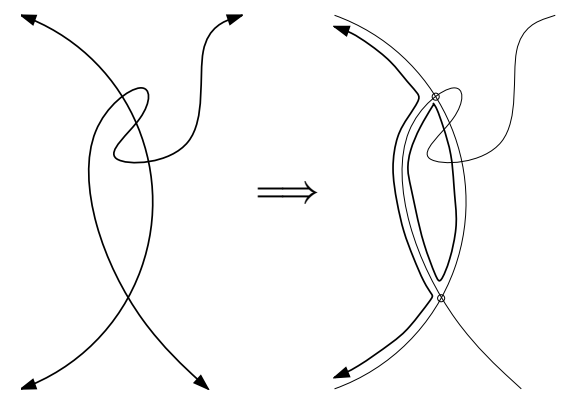

Figure 19: Compression of a disk

to it. And since $B_{2}^{\prime}$ does not intersect $B_{2}$ at all, it is descent equivalent to it. So both $B_{1}$ and $B_{2}$ are descent equivalent to $B_{2}^{\prime}$, which is a contradiction.

We assume from now on that all components of the intersection of $B_{1}, B_{2}$ are of the remaining types. Then $B_{1}, B_{2}$ are vertical bands and not spheres or disks. Furthermore their boundaries are essential circles in $\partial M$, otherwise, we could cap them to form disks or spheres.

Case $\mathbf{a} 3$ and $\mathbf{m} 3$ The curve is a circle in $B_{1}$ such that it and one component of $\partial B_{1}$ bound an annulus $A$. Then we can find a curve $C$ such that $A$ does not contain any other component of the intersection. Then $C$ divides $B_{2}$ into an annulus $A^{\prime}$ and another component $B^{\prime}$, which is either another annulus or a Möbius strip. Indeed, $B_{2}$ must be of type either a3 or $\mathrm{m} 3$ since if it was of type $\mathrm{m} 5$, then $B_{1}$ would also be of type m5. One of $A^{\prime}$ or $B^{\prime}$ in union with $A$ forms a vertical band $B$, which is essential since $\partial B$ is made of circles essential in $\partial M$. And after isotopy, $B$ has a simpler intersection with both $B_{1}$ and $B_{2}$ than they do each other, so it is descent equivalent to both, which is a contradiction.

Case m5 Let $C$ be isotopic to the core of $B_{1}$, so it is also isotopic to the core of $B_{2}$, which must also be of type m5. Then it cuts each into an annulus, and after an isotopy, the two together form an essential vertical annulus $A$ with simpler intersection with both $B_{1}$ and $B_{2}$ than they do each other, so it is descent equivalent to both, which is a contradiction.

Case a4 and $\mathbf{m} 4$ Finally, the intersection consists only of vertical arcs, so a regular neighborhood of the union of the two essential vertical bands has at its boundary vertical bands, each disjoint from either $B_{1}$ or $B_{2}$. If any band is essential, then it is descent equivalent to both $B_{1}$ and $B_{2}$, which is a contradiction, but if none is essential, then one of them separates $B_{1}$ and $B_{2}$ from the link, which contradicts the fact that they are essential. 


\section{Twisted link diagrams}

Theorem 2 will follow from the following lemma.

Lemma 1 There is a bijection between each of the following:

(1) Ambient isotopy equivalence classes of links in stable oriented thickenings.

(2) Reidemeister equivalence classes of link diagrams in stable surfaces.

(3) Reidemeister equivalence classes of abstract links.

(4) Reidemeister equivalence classes of twisted link diagrams.

Figure 20 illustrates the process of creating a link diagram from a link in a thickened
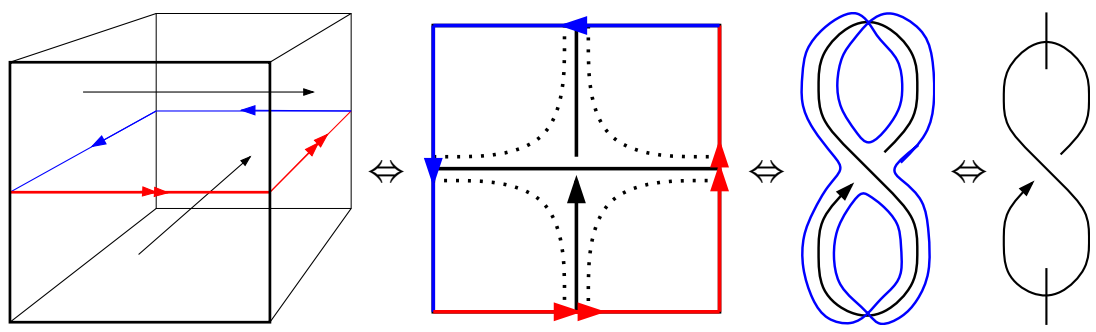

Figure 20: Creating a diagram for a twisted link

Klein bottle. The first step is a regular projection of the link to create a link diagram in the zero-section. The second step creates an abstract link diagram in a regular neighborhood of the link diagram in the surface. The third step immerses this diagram in the plane, and decorates the result with virtual crossings and bars.

Proof $(1) \Leftrightarrow(2)$ Fix an oriented thickening of a closed surface and consider the surface as the zero-section of the oriented thickening. By Sard's theorem, the set of embeddings of a link in the manifold that have regular projections to the surface is dense in the set of all ambient-isotopic embeddings that form a link. The surface projection defines a resolution of the double points of the projection as follows. Given the fiber over a neighborhood about the double point, if both domain strands are on the same side of the neighborhood or on the neighborhood, the image of the strand whose doubled point is closer to the boundary of its side has its $\eta$ vector towards that boundary while the other strand has its $\bar{\eta}$ vector towards the other boundary. And if the strands are on different sides, their $\eta$ 's are towards their side's boundary. Define a separation of the surface projection from the resolution as $\omega=\bar{\tau} \times \eta, \bar{\omega}=\tau \times \bar{\eta}$. 
On the other hand, given a link surface diagram, choose an oriented thickening of the surface. Define a resolution of the surface projection from the separation as $\eta=\omega \times \bar{\tau}, \bar{\eta}=\bar{\omega} \times \tau$, and resolve the double point by the vectors. If the thickening with the opposite orientation had been chosen, the two links in oriented thickenings are related by an orientation-preserving homeomorphism.

By Hudson and Zeeman [7], two embeddings of links in an oriented thickening are ambient isotopic if and only if they are ambient isotopic by linear moves in arbitrary small neighborhoods. Then we may choose neighborhoods of curves such that their projections to the surface are generic curves whose intersections are in disks. Then we are in the classical case, and have that links that have regular projections are ambient isotopic in the manifold if and only if their link surface diagrams are Reidemeister equivalent.

There exists a destabilization of the link in oriented thickening along a topologically vertical band if and only if there is a destabilization of the link surface diagram possibly preceded by a sequence of Reidemeister moves. Indeed, a vertical band exists as the fiber over a simple closed path in the projection surface. And if the band is only topologically vertical, it can be made vertical through an isotopy of the link that corresponds to a sequence of Reidemeister moves because it is isotopic to a vertical band.

(2) $\Leftrightarrow$ (3) Given a link surface diagram, a regular neighborhood of the link is an abstract link diagram. Given an abstract link diagram, fill in disks along its boundary components to obtain a cellular link surface diagram, then stabilize the surface to obtain the original link surface diagram.

Reidemeister moves on link surface diagrams are done in disk neighborhoods obtained after stable isotopy of the link brings the strands together. And Reidemeister moves on abstract link diagrams are done in disk neighborhoods obtained by bringing the strands together with homeomorphisms of the boundary.

(3) $\Leftrightarrow$ (4) Obtain an abstract link diagram from a twisted link diagram, as follows. Thicken the plane into an oriented $\mathbb{R}^{2} \times I$ with the diagram in the zero-section. Choose nonintersecting disk neighborhoods of each classical crossing that contain no other part of the diagram, and choose nonintersecting connected neighborhoods in the boundary of each disk for each intersection of the arcs through that crossing with the disk boundary. Define neighborhoods of each arc between the disk neighborhoods of the crossings at its ends by continuously sliding an interval from one neighborhood of the arc's intersection with a crossing's disk neighborhood to the other along the arc such that the interval is always centered on the arc and normal to it, and passes through the normal to the zero-section if and only if there is a bar at that point on the arc. The 
width of the interval can be adjusted during its slide so that the neighborhood it defines does not intersect with either another arc neighborhood or a disk neighborhood of a classical crossing. Move the arc neighborhoods by a small amount so that they do not intersect one another into one of the two ways shown in Figure 21. Different choices of

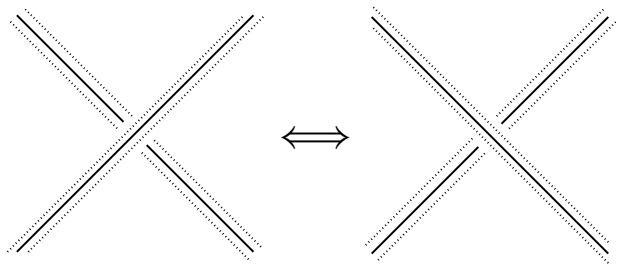

Figure 21: Two ways to resolve a virtual crossing

movement of the intervals defining the arc neighborhoods and of direction of turn of the interval as it slides along the arc yield homeomorphic abstract link diagrams although they may produce different embeddings of that diagram in the thickened plane.

Suppose two link diagrams differ by an extended Reidemeister move. If the move is classical, then the equivalent classical move exists on the abstract link diagram. If the move is virtual or twisted, then the embedding is changed in some version of what is shown in Figure 22 but the abstract link diagram is unchanged. Note that V3, V4, T1,

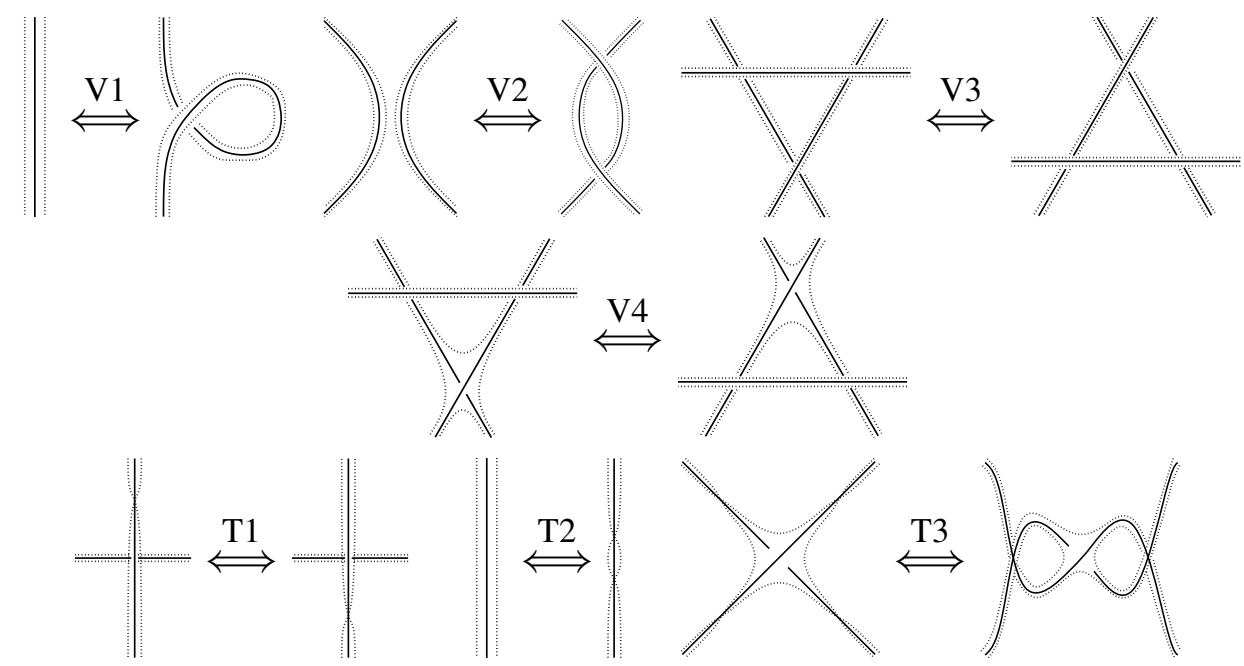

Figure 22: Virtual and twisted changes to embeddings

and $\mathrm{T} 3$ are isotopies of the embedding, while V1 and T2 are equivalent up to twist direction.

Algebraic 83 Geometric Topology, Volume 8 (2008) 
Obtain a twisted link diagram from an abstract link diagram as follows. Embed the abstract link diagram in $S^{2} \times I$ such that each crossing has a disk neighborhood in $S^{2}$, and that the projection of the embedding to $S^{2}$ is an immersion. On the projected diagram, draw crossings in the over-under form depending on the resolution of the abstract link crossings. Draw each intersection of two projected arcs as a virtual crossing. Draw each intercomponent intersection of the projection of the two boundary components of the neighborhood of each arc as bars on the arc. Figure 23 shows the

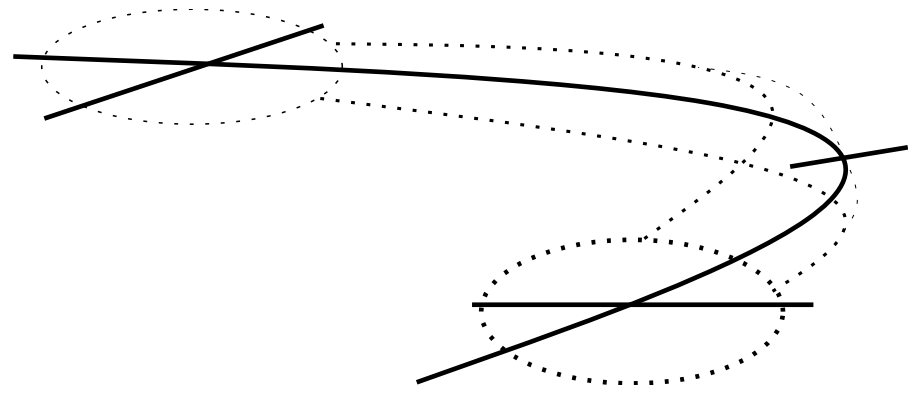

Figure 23: Arc boundary intersection giving rise to a bar

projection of an arc and the boundary components, and draws one bar on the arc for the single intercomponent intersection of thee two boundary components.

Suppose two different embeddings $g, g^{\prime}$ of the same abstract link give rise to different twisted link diagrams. We can change $g$ by embedding each neighborhood of a crossing with the opposite rotation of the arcs to make them match that of $g^{\prime}$, which corresponds to a series of T3 moves. Figure 24 shows the effect of performing a T3 move on both

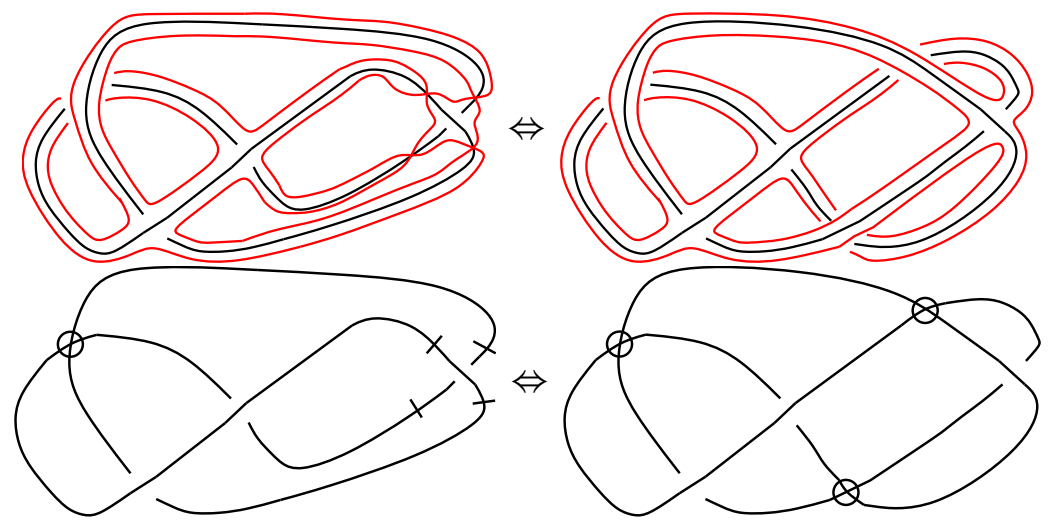

Figure 24: Performing a T3 move

an embedding of an abstract link and on the corresponding twisted link diagram. We 
can change $g$ by an isotopy of $S^{2}$ to make its neighborhoods of crossings including the arcs correspond to that of $g^{\prime}$, which corresponds to a series of V4 moves. For each arc neighborhood in turn, we can change $g$ by a homotopy until the arc coincides with that of $g^{\prime}$, which corresponds to a finite sequence of V1-4 and T1 moves, and we can then change $g$ 's embedding of the arc neighborhood itself so that it matches that of $g^{\prime}$, which corresponds to a finite sequence of T1-2 moves.

Suppose two abstract links differ by a single R1-3 move. By the above, we can choose embeddings of these links that coincide outside of a disk neighborhood containing the crossings and edges involved. Then the twisted link diagrams will differ by the corresponding R1-3 move.

Since the projection of a link is a generic immersion of curves in the surface homeomorphic to the zero-section, then projections of a link in an oriented thickening are classified by intersigned Gauss codes. By adding a writhe sign to an intersigned Gauss code, we obtain an intersigned link code, and we then obtain the following:

Corollary 2 Reidemeister equivalence classes of link diagrams in stable surfaces correspond to Reidemeister equivalence classes of intersigned link codes.

\section{The twisted Jones polynomial is an invariant}

A smoothing of a real crossing of a link diagram is a transformation of one of two kinds labeled "a" and "b" as shown in Figure 25. A state of a link diagram is a collection
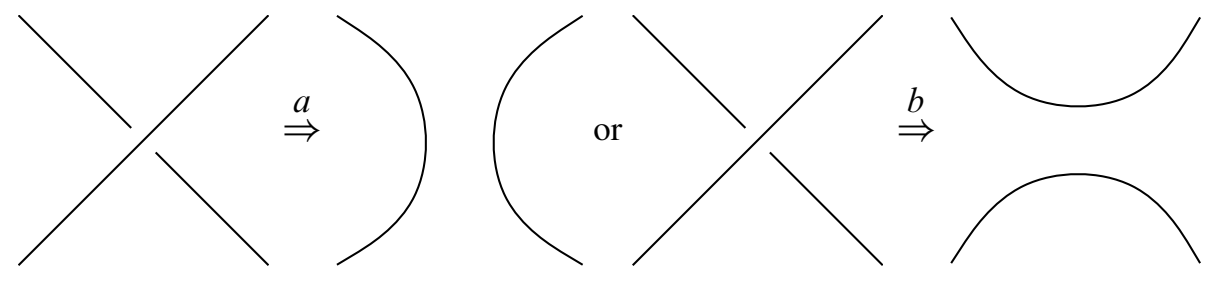

Figure 25: Two possible smoothings of a crossing

of smoothings of all its crossings. The bracket polynomial of a link diagram $D$ is an element of $\mathbb{Z}\left[A^{ \pm 1}, M\right]$ defined from the possible states $\mathcal{S}(D)$ of $D$ by

$$
\langle D\rangle=\sum_{S \in \mathcal{S}(D)} A^{a(S)-b(S)}\left(-A^{-2}-A^{2}\right)^{c(S)} M^{d(S)}
$$


where:

- $a(S)$ is the number of $a$-smoothings,

- $b(S)$ is the number of $b$-smoothings,

- $c(S)$ is the number of circles with an even number of bars, and

- $d(S)$ is the number of circles with an odd number of bars.

The twisted Jones polynomial of a link diagram $D$ is an element of $\mathbb{Z}\left[A^{ \pm 1}, M\right]$ calculated as:

$$
V_{D}(A, M)=(-A)^{-3 w(D)}\langle D\rangle .
$$

We prove that the extension to the Jones polynomial defined above is an invariant of links in oriented thickenings, and that it distinguishes a class of links in oriented thickenings from virtual links.

Proof of Theorem 3 The bracket polynomial of link diagrams in oriented thickenings is equivalent to that defined by the relations:

(1) $\langle\varnothing\rangle=1$,
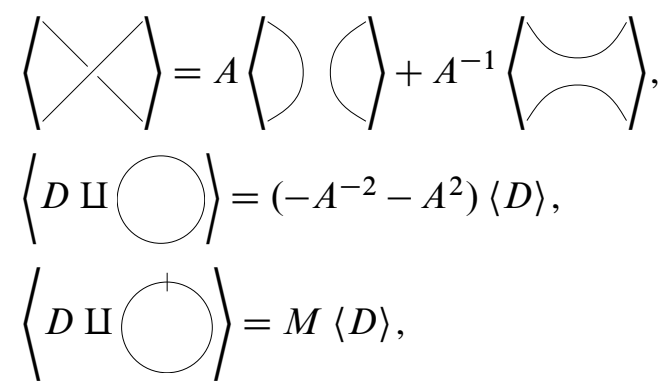

$$
\langle\mid\rangle=\langle\neq\rangle \text {. }
$$

This can be seen by first using relation (2) to smooth the crossings of a diagram $D$ in all possible ways, so that each final diagram of circles corresponding to one of the states of $D$. Then relation (5) allows each circle to be reduced to either 0 or 1 bars. Finally, the other relations allow the calculation of the bracket polynomial.

Invariance with respect to R1-3 and V1-4 is immediately due to that of the Jones polynomial. Invariance with respect to $\mathrm{T} 1$ is immediate since it involves no real 
crossings. Invariance with respect to $\mathrm{T} 2$ is shown by relation (5). Then the twisted Jones polynomial is invariant with respect to move T3 by the calculation:

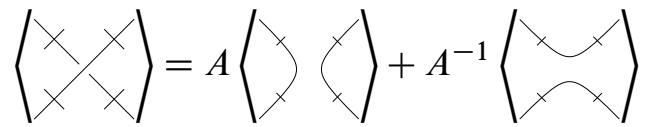

$$
\begin{aligned}
& =A \curlywedge(\rangle+A^{-1} \backslash \\
& =A\left\langle\oint \oint+A^{-1} \oint \oint\right\rangle \quad \text { by }(\mathrm{V} 1, \mathrm{~V} 2) \\
& =\langle\varnothing\rangle \text {. }
\end{aligned}
$$

So the twisted Jones polynomial is an invariant of links in oriented thickenings.

Since virtual links do not have bars on their edges, then their states will not have any circles with an odd number of bars, and so their twisted Jones polynomials will be in $\mathbb{Z}\left[A^{ \pm 1}\right]$. And since the $\left(-A^{-2}-A^{2}\right)$ term is raised to the number of circles in a state, the twisted Jones polynomial will factor into a product of $\left(-A^{-2}-A^{2}\right)$ and the Jones polynomial of the diagram.

The Jones polynomial can be extended to links in oriented thickenings by the device of ignoring bars on edges. This invariant corresponds to setting $M=-A^{-2}-A^{2}$ in the new polynomial and then dividing the result by $-A^{-2}-A^{2}$.

The onefoil shown in Figure 3 has twisted Jones polynomial:

$$
V_{\text {Onefoil }}(A, M)=A^{-6}+\left(1-M^{2}\right) A^{-2}
$$

so it is not a virtual knot.

\section{Twisted Jones of two-colorable diagrams}

The knot $K$ in Figure 6 has twisted Jones polynomial:

$$
V_{K}(A, M)=\left(-A^{-2}-A^{2}\right)\left(A^{-4}+A^{-6}-A^{-10}\right)
$$

which is a product of $\left(-A^{-2}-A^{2}\right)$ and of the Jones polynomial for the knot. But this knot is in the projective plane and cannot be moved to an affine subset of this space, so it is not a classical knot [3]. We will see that the knot's twisted Jones polynomial factors in this manner because it has a two-colorable diagram. 
Proof of Theorem 4 Let $D$ be a two-colored diagram for a link. This two-coloring partitions the faces of $D$ into two sets of circles each of one of the two colors and such that each crossing of the diagram will separate a disk neighborhood of that crossing into two pairs of regions that are opposite one another with respect to the crossing and that contain like-colored parts of the faces of the diagram. Figure 26 shows this

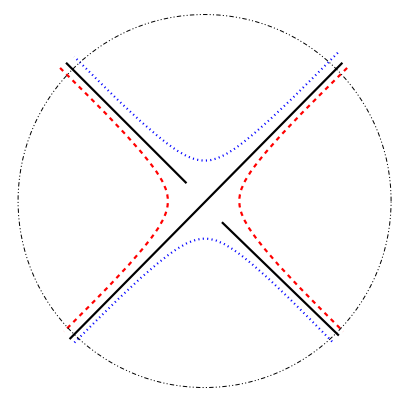

Figure 26: A crossing of a two-colored diagram

situation for an arbitrary crossing. In the figure, the parts of faces are drawn either dotted or dashed to represent the two colors while the boundary of the disk is drawn with a dash-dot-dotted pattern. The two possible smoothings of each crossing of a diagram also pair opposite regions of the crossing. In the figure, the dashed lines are the " $a$ " smoothing while the dotted lines are the " $b$ " smoothing. Then the faces of one color of $D$ are the circles of the state of $D$ whose smoothings pair the corresponding two opposite regions of each crossing, and the faces of the other color are the circles of the complementary state of $D$. Since the circles of each of these states correspond to faces of a diagram and that by the Jordan curve theorem the faces cross the link diagram an even number of times at bars, then the circles have an even number of bars.

All other pairs of states of $D$ can be obtained from the above pair of states by taking the opposite smoothings at some subset of the crossings. Changing a state at a crossing corresponds to putting bars on all the edges coming into that crossing, and the effect of this change on the faces is show in Figure 27. In the figure, the diagram on the left shows " $a$ " smoothing as the dashed lines pairing the left and right regions, while the " $b$ " smoothing is the dotted line pairing the top and bottom regions, and the diagram on the right has the opposite pairings. While adding the bars changes the paths that form the circles of the new state, these circles still have an even number of bars. Therefore all the states of $D$ have circles with an even number of bars on the circles. Then the twisted Jones polynomial for this link will not have any $M$ term, and will be divisible by $-A^{-2}-A^{2}$. 


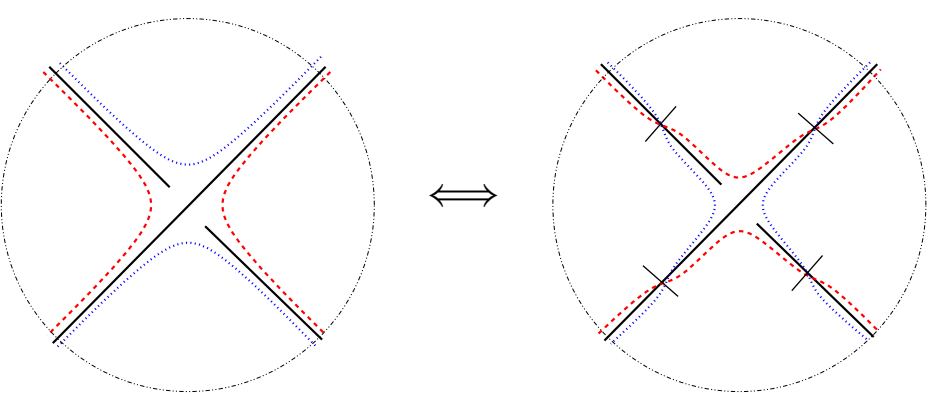

Figure 27: Changing the smoothings at a crossing

\section{The twisted link group is an invariant}

A Wirtinger presentation of a group is a finite presentation whose relators are of the form $a^{-1} c^{-1} b c$ for any three generators $a, b, c$ of the presentation. When $c=b$, the relation becomes the identification $a^{-1} b$, so identification relations are admissible in a Wirtinger presentation.

The group of a link diagram $\Pi L$ is a group derived from a link diagram $L$, and has a Wirtinger presentation that has one generator for every arc of the diagram between undercrossings, and one relation for every crossing. Figure 28 shows three generators of

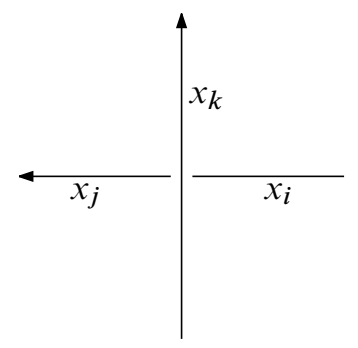

Figure 28: Generators at a crossing for a formal presentation

a formal Wirtinger presentation obtained from a diagram of a virtual link at a crossing. These generators have the relation:

$$
x_{j}=x_{k}^{-1} x_{i} x_{k}
$$

The group of a link diagram is also called the upper group of the diagram and may be denoted $\Pi^{u} L$. The lower group $\Pi^{l} L$ of a link diagram has one generator for every arc of the diagram between overcrossings, and again one relation for every crossing defined analogously to that of the upper group.

Algebraic $8 \mathcal{G}$ Geometric Topology, Volume 8 (2008) 
The twisted link group of a diagram $\widetilde{\Pi} L$ is a group with a Wirtinger presentation that has a generator for each side of each end of an edge. Figure 29 shows eight generators

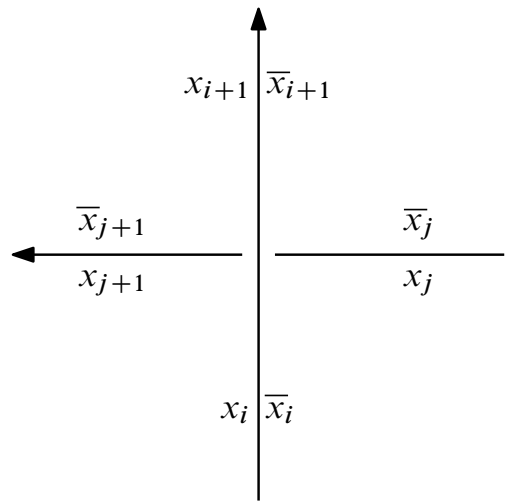

Figure 29: Generators at a crossing for a twisted presentation

obtained from a diagram of a link at a crossing. These generators have the four crossing relations:

$$
\begin{array}{llrl}
x_{i+1} & =x_{i}, & x_{j+1} & =x_{i}^{-1} x_{j} x_{i}, \\
\bar{x}_{i+1} & =\bar{x}_{j}^{-1} \bar{x}_{i} \bar{x}_{j}, & \bar{x}_{j+1} & =\bar{x}_{j} .
\end{array}
$$

The four generators of an edge have two relations depending on the parity of the number of bars on the edge. Figure 30 shows the four generators of an edge.

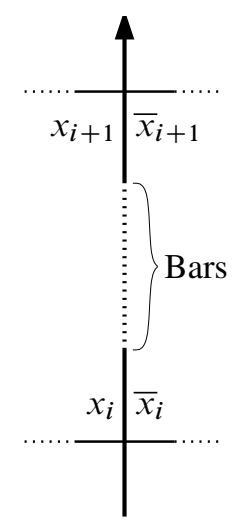

Figure 30: Generators on an edge for a twisted presentation 
When an edge has an even number of bars between two crossings, we have the relations:

$$
x_{i+1}=x_{i}, \quad \bar{x}_{i+1}=\bar{x}_{i},
$$

and when there is an odd number of bars on the arc, we have the relations:

$$
x_{i+1}=\bar{x}_{i}, \quad \bar{x}_{i+1}=x_{i}
$$

Proof of Theorem 5 Note that performing moves V1-4 and T1-2 on a link diagram yield a link diagram with an identical presentation. Invariance with respect to moves R1-3 is the same as for classical link diagrams since neither virtual crossings nor bars are involved. Finally, invariance with respect to T3 is as follows. Figure 31 shows half
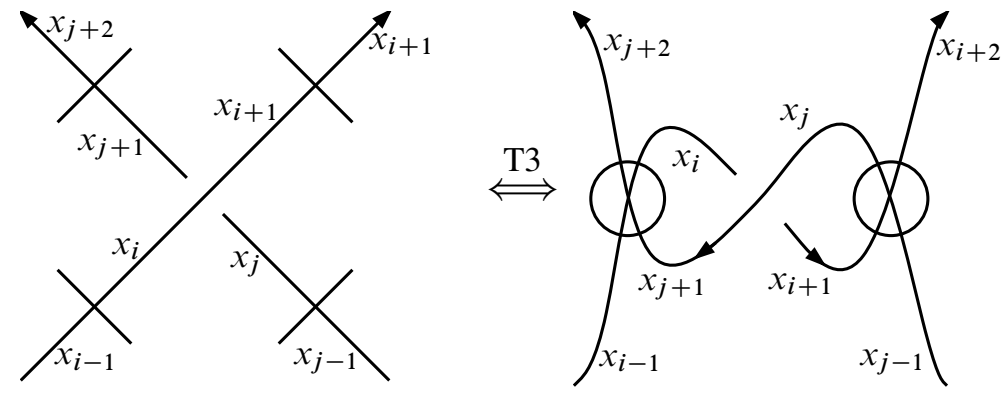

Figure 31: The generators in move T3

of the generators on the edges of the two diagrams. Then the relations given by the crossing are:

$$
\begin{array}{ll}
\text { On the left } & \text { On the right } \\
x_{j+1}^{-1} x_{i}^{-1} x_{j} x_{i} & x_{j+1}^{-1} x_{i}^{-1} x_{j} x_{i} \\
x_{i+1}^{-1} x_{i} & x_{i+1}^{-1} x_{i}
\end{array}
$$

which are identical. Doing the same for the other half of the generators shows the corresponding equivalence.

\section{References}

[1] M Bourgoin, Classifying immersed curves, in preparation

[2] M Bourgoin, On the fundamental group of a virtual link, in preparation

[3] Y V Drobotukhina, An analogue of the Jones polynomial for links in $\mathbf{R P}^{3}$ and a generalization of the Kauffman-Murasugi theorem, Algebra i Analiz 2 (1990) 171-191 MR1073213 
[4] Y V Drobotukhina, Classification of projective Montesinos links, Algebra i Analiz 3 (1991) 118-130 MR1120843

[5] Y V Drobotukhina, Classification of links in $\mathbf{R P}^{3}$ with at most six crossings, from: "Topology of manifolds and varieties", Adv. Soviet Math. 18, Amer. Math. Soc. (1994) 87-121 MR1296890

[6] J L Gross, T W Tucker, Topological graph theory, Wiley-Interscience Series in Discrete Math. and Optimization, John Wiley \& Sons, New York (1987) MR898434

[7] J F P Hudson, E C Zeeman, On combinatorial isotopy, Inst. Hautes Études Sci. Publ. Math. (1964) 69-94 MR0163318

[8] V F R Jones, A polynomial invariant for knots via von Neumann algebras, Bull. Amer. Math. Soc. (N.S.) 12 (1985) 103-111 MR766964

[9] N Kamada, On the Jones polynomials of checkerboard colorable virtual links, Osaka J. Math. 39 (2002) 325-333 MR1914297

[10] N Kamada, S Kamada, Abstract link diagrams and virtual knots, J. Knot Theory Ramifications 9 (2000) 93-106 MR1749502

[11] L H Kauffman, State models and the Jones polynomial, Topology 26 (1987) 395-407 MR899057

[12] L H Kauffman, Virtual knot theory, European J. Combin. 20 (1999) 663-690 MR1721925

[13] G Kuperberg, What is a virtual link?, Algebr. Geom. Topol. 3 (2003) 587-591 MR1997331

[14] V O Manturov, Curves on surfaces, virtual knots, and the Jones-Kauffman polynomial, Dokl. Akad. Nauk 390 (2003) 155-157 MR2003612

[15] M Mroczkowski, Diagrammatic unknotting of knots and links in the projective space, J. Knot Theory Ramifications 12 (2003) 637-651 MR1999636

[16] M Mroczkowski, Polynomial invariants of links in the projective space, Fund. Math. 184 (2004) 223-267 MR2128052

[17] S Nelson, Unknotting virtual knots with Gauss diagram forbidden moves, J. Knot Theory Ramifications 10 (2001) 931-935 MR1840276

[18] DS Silver, S G Williams, Virtual knot groups, from: “Knots in Hellas '98 (Delphi)", Ser. Knots Everything 24, World Sci. Publ., River Edge, NJ (2000) 440-451 MR1865722

Department of Mathematics, Brandeis University

415 South Street, MS 050, Waltham, MA 02454, USA

mob@brandeis.edu

http://people.brandeis.edu/ mob/

Received: 10 August 2006

Algebraic $\&$ Geometric Topology, Volume 8 (2008) 